\title{
Development of a novel therapy in virtual motor rehabilitation after severe Guillain-Barré symptomatology
}

\author{
Sergio Albiol-Pérez ${ }^{1}$, José-Antonio Gil-Gómez ${ }^{3}$ \\ ${ }^{1}$ Dpto. de Informática e Ingeniería de Sistemas, \\ Universidad de Zaragoza, \\ Ciudad Escolar s/n 44003, Teruel, SPAIN \\ ${ }^{3}$ Instituto Universitario de Automática e Informática \\ Industrial, Universitat Politècnica de València, \\ C. Vera S/N, 46022, Valencia, SPAIN
}

\author{
Mercedes Forcano-García ${ }^{2}, M^{a}$ Teresa Muñoz- \\ Tomás ${ }^{2}$, Pilar Manzano-Hernández ${ }^{2}$, Sofía Solsona- \\ Hernández $^{2}$ \\ ${ }^{2}$ Hospital S. José, Av. Zaragoza 16, 44001, Teruel, SPAIN
}

\begin{abstract}
Guillain-Barré syndrome is an inflammatory polyradiculoneuropathy where the etiology is not clear. Some of the most important problems in this type of syndrome are balance and gait disorders. Traditional rehabilitation includes exercises such as proper limb positioning, posture, orthotics, and activities that patients carry out in daily living (ADL). Novel therapies based on Virtual Motor Rehabilitation (VMR) are alternative treatments to increase the patient's motor recovery. In this paper, we proposed a virtual rehabilitation system (ABAR) to increase the results in static and dynamic balance for Guillain-Barré patients. The results of the clinical test show that the use of the ABAR system improves balance, postural control, and gait in Guillain-Barré patients.
\end{abstract}

Keywords; Virtual Motor Rehabilitation; Guillain Barre; Balance; Balance Control; Gait.

\section{INTRODUCTION}

Guillain-Barré syndrome (GBS) is an acute monophasic illness that causes a rapid, progressive polyneuropathy with weakness or paralysis. The cardinal clinical features [1],[2] of GBS are progressive, mostly symmetric muscle weakness and absent or depressed deep tendon reflexes. The weakness can vary from mild difficulty with walking to nearly complete paralysis of all extremity, facial, respiratory, and bulbar muscles. Severe respiratory muscle weakness necessitating ventilatory support develops in about 30 percent of patients, and dysautonomia occurs in 70 percent of patients. GBS usually progresses over a period of about two weeks.

GBS is a heterogeneous syndrome with several variant forms. Acute inflammatory demyelinating polyneuropathy (AIDP) is the most common variant of GBS in North America, Europe, and most of the developed world [3]. The Miller Fisher syndrome (MFS) is a GBS variant that is characterized by ophthalmoplegia with ataxia and areflexia [4]. Axonal forms of GBS include acute motor axonal neuropathy (AMAN), which is most common in Japan and China, and acute motor and sensory axonal neuropathy (AMSAN). The initial diagnosis of GBS is based on the clinical presentation. The diagnosis is confirmed by cerebrospinal fluid (CSF) analysis and clinical neurophysiology studies. Therefore, these studies should be performed in all patients with suspected GBS.

\section{RELATED WORK}

Due to this muscle weakness, some of the main problems that patients have are postural control, high rates of fatigue [5], or even paralysis. Balance Disorders are one of the most important risks of falls in diseases such as Guillain-Barré syndrome. Balance rehabilitation is one of the most important therapeutic treatments for patients with gait disorders.

Acute-phase rehabilitation should include an individualized program of gentle strengthening, involving isometric, isotonic, isokinetic, and manual resistive and progressive resistive exercises [6]. Rehabilitation should emphasize proper limb positioning, posture, and orthotics as well as nutrition. A device to help with communication may be necessary. After the acute phase, disabled patients should be treated by a multidisciplinary rehabilitation team [7]. An exercise program may be beneficial for persistent fatigue [6].

There have been few systematic studies on the efficacy of rehabilitation, including physical therapy for patients with GBS. In 2004, a Cochrane Systematic Review was published on exercise in peripheral neuropathy [8]. The review found inadequate evidence to evaluate the effects of exercise on disability in peripheral neuropathy, but it found evidence that strengthening exercises moderately improve muscle strength. Only one systematic exercise study has been published on patients with GBS, where 16 patients with relatively good recovery from GBS bicycled 3 times per week for 12 weeks [9]. These patients were in a stable phase. Self-reported fatigue decreased and physical fitness improved significantly with this training regime. Two case reports also support the notion that endurance exercise training could improve aerobic capacity and strength in GBS with residual deficits [10]. In later stages, exercise programs may be helpful in combatting fatigue, increasing muscle strength and improving cardio-pulmonary function [11]. However, scientific evidence is scarce. 
Incomplete recovery with residual signs has been reported in proportion $50 \%$ of patients at 1-2 years after onset [12]. This partial recovery may be a significant cause of long-term disability.

Balance rehabilitation is one of the most important therapeutic techniques in patients that suffer gait problems. This therapeutic process focuses on restoring balance, where patients must simulate, as soon as possible, the different movements and activities that they carried out in daily living (ADL).

Traditional methods for training balance can be repetitive, boring, and inaccurate, which sometimes causes low motivation and low adherence to the treatment, limiting the benefits. Posturography assessment, which is based on force platforms, can estimate the patient's weight distribution by means of pressure sensors [13]. These types of systems are alternative feedback devices to work balance patients, but the main drawbacks are the high cost and the large area needed to test the rehabilitation process.

In the last few years, the use of Virtual Reality in traditional rehabilitation to increase balance and postural control is a reality [14],[15]. Using devices in conjunction with virtual environments (VE) provides conditions where patients interact by simulating traditional methods and obtaining significant results. In [16], the authors concluded that the use of VE that are similar to the "real" world with challenges that emulate functional and real behavior increase the results of traditional rehabilitation.

Novel and low-cost force platform system, with visual biofeedback systems, such as the Nintendo ${ }^{\circledR}$ Wii Balance Board are an interesting option to obtain balance improvements in virtual rehabilitation therapies [17][18]. The main drawback of these systems is due to the use of commercial games in the virtual sessions because of their complexity. To solve this, it is necessary to create new tools that are specifically designed for patients with balance disorders, increasing the potential of the patient's motor recovery [19].

The purpose of this study was to evaluate static and dynamic balance control in two Guillain-Barré patients. For this purpose, we used a novel and customizable tool, the Active Balance Rehabilitation system (ABAR) [20], which is specifically designed for patients with balance and gait problems. This novel therapy will improve the effectiveness of rehabilitation and the results of the balance test, increasing patient motivation and involvement in the rehabilitation process.

\section{METHODS}

\section{A. Participants}

Subject 1 was a 54-year-old man, 5 feet and 74 inches in height, with a body-mass index of 24.65 , a weight of $75.5 \mathrm{~kg}$, and a calf circumference of $35 \mathrm{~cm}$. On admission, he did not experience any fall, but he needed the assistance of a walker. The initial Barthel Index was 60 points and without existence of cognitive impairment (MEC-Lobo [21]) > 23 .
TABLE I.

CHARACTERISTICS OF PATIENTS

\begin{tabular}{lcc}
\hline \hline \multicolumn{1}{c}{ Issue } & Patient one & Patient two \\
\hline Gender & Male & Male \\
\hline Age (years) & 54 & 33 \\
\hline Time since injury (months) & 5 & 4 \\
\hline Weight & 75.5 & 94 \\
\hline Height (ft) & 5.74 & 5.54 \\
\hline
\end{tabular}

Subject 2 was a 33-year-old man, 5 feet and 54 inches in height, with a body-mass index of 32.91, a weight of $94 \mathrm{~kg}$, and a calf circumference of $42 \mathrm{~cm}$. On admission, he had suffered previous falls (specifically ten falls), but he did not need help to walk. The initial Barthel Index was 95 points and without existence of cognitive impairment (MEC-Lobo [21]) > 23. The characteristics of patients are showed in Table 1. Instrumentation

ABAR is composed of a specific Virtual Environment (VE) that was designed and developed by clinical specialists in rehabilitation, to increase balance and postural control in different pathologies such as: Acquired Brain Injury, Multiple Sclerosis, or Guillain-Barré syndrome.

The main goals that we have achieved are: 1) the establishment of a valid and adaptive system in the balance retraining of the patient, reducing the risk of falls and fractures; 2) the development of a tool that reinforces the patient's motivation during the rehabilitation process; 3) the use of visual biofeedback, that shows the performance of the balance and postural control obtained in each of the sessions of the rehabilitation process; 4) improvements in gait control, muscle tone, flexibility, and stability in sitting and standing, compensating vestibular disorders or other features that often affect the stability in Guillain-Barré patients; 5) the use of Virtual Rehabilitation techniques at home after the rehabilitation sessions to maintain the improvements in the acute and sub-acute stages; 6) the customization of treatments with increasing difficulty, which are adjusted to the specific situation of each patient.

The system has two levels of difficulty: low and medium. The low level has two VE to train antero-posterior and mediolateral weight transferences in the sitting position. In these virtual environments, the physiotherapist can modify the level of difficulty easily because there are specific parameters such as: target speed, time that the target is shown on the TV, or even the number of sessions, session time, and a specific parameter where the specialist can modify the rest period between virtual sessions.

The medium level has four different VE in order to obtain specific movements and weight transferences. The main purpose of this type of therapy is to rehabilitate static and dynamic balance in the standing position. In static balance, the system requires that patients perform different weighttransferences in the standing and sitting position (medio-lateral and antero-posterior weight-transferences). In dynamic balance, ABAR forces the patient to make different movements in the standing position (to step on the WBB or sitting-standing movements). Movements are showed in Table 2. 
TABLE II. POSITIONS / MOVEMENTS IN ABAR SYSTEM

\begin{tabular}{l} 
SITTING POSITION \\
\hline \hline Movement positions \\
$\begin{array}{c}\text { The patient performs weight } \\
\text { transferences in the medio-lateral or } \\
\text { antero-posterior direction in the sitting } \\
\text { position. }\end{array}$ \\
$\begin{array}{c}\text { The patient performs medio-lateral } \\
\text { weight transferences in the sitting } \\
\text { position. }\end{array}$
\end{tabular}

\section{STANDING POSITION}

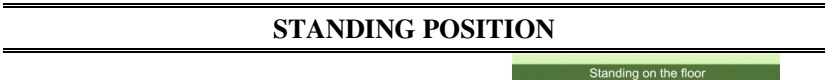

The patient moves his feet from left to right by shifting his weight in the standing position.

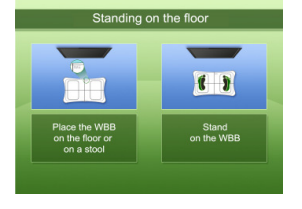

The patient moves his feet in the tandem position by shifting his weight from foot to foot, forwards and backwards.

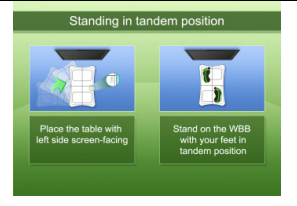

The patient moves in the standing or sitting position.

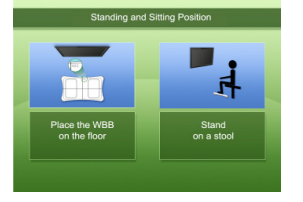

The patient makes a step with his right or left foot onto the WBB in the standing position.

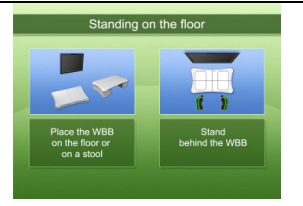

\section{PROCEDURE}

The study was carried out in a small metropolitan hospital. The Guillain-Barré patients performed a total of 20 sessions using ABAR. The length of the sessions for patients was around 30 minutes. We tested different clinical tests in two periods of time (Initial and Final Evaluation).

In both patients, the therapist measured different clinical tests related to Cognitive impairment such as: an adapted and validated version of the Mini-Mental state examination (MECLobo) [21]; Activities of Daily living (ADL): Barthel index [22], Lawton's Philadelphia Geriatric Center Morale Scale (PGCMS) [23], Charlson Comorbidity Index (CCI) [24].

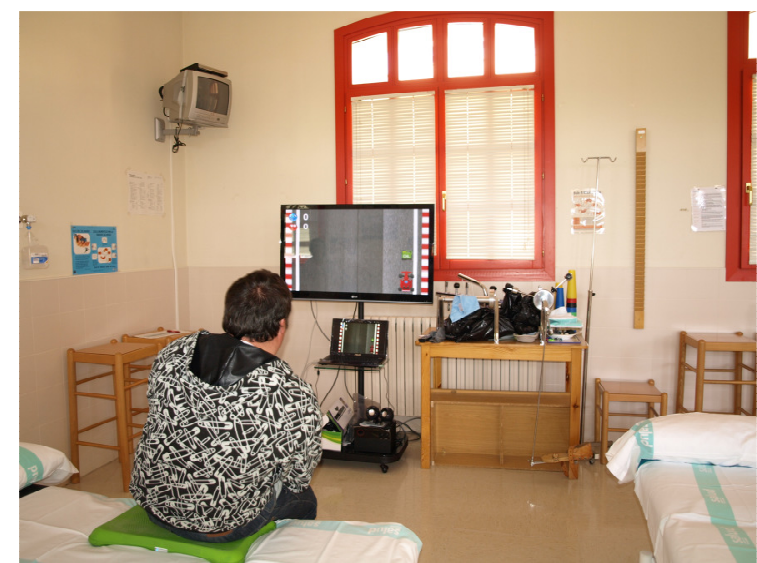

Fig. 1. Weight transferences in the sitting position using the ABAR system.

Other clinical tests that we measured were based on Static balance: Unipedal Stance Time (UST) [25], Anterior Reach Test (ART) [26], and Dynamic balance: Berg Balance Scale Test (BBS) [27], the Time "Up and Go" Test (TUG) [28], the Timed 10-Meter Walking Test (10 MWT) [29] , Tinetti Test (TT) [30] and 30-second Sit-to-Stand Test (30SST) [31].

At the end of the first session, we applied Suitability Evaluation Questionnaire (SEQ) in order to obtain biofeedback information about the virtual treatment.

\section{RESULTS}

Eight months after the injury, Subject 1 achieved independent walking under the supervision of the therapist. He was able to go up, and down stairs and to walk on the slope, with an increase in muscle mass, improving joint range in both shoulders; however there were disturbances in upper $\operatorname{limb}$ motor coordination.

In contrast, six months after the injury, Subject 2 performed independent walking without any type of assistance. He was able to go up, down stairs and to walk on the slope easily, maintaining hypesthesia in fingers, the soles and the back of the feet, obtaining muscle tone in the left ankle. The results obtained in the two periods of time (Initial and Final Evaluation) are shown in Figure 2 and Figure 3:

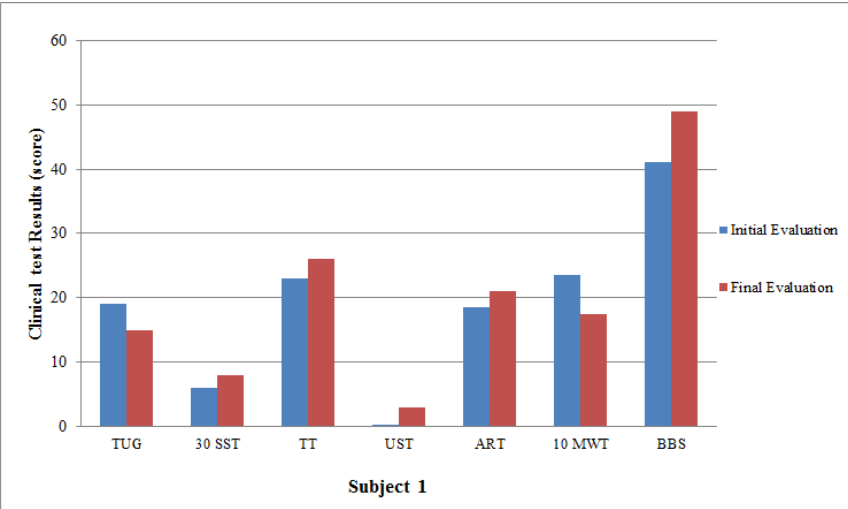

Fig. 2. Clinical test Results of subject 1 using the ABAR system. 


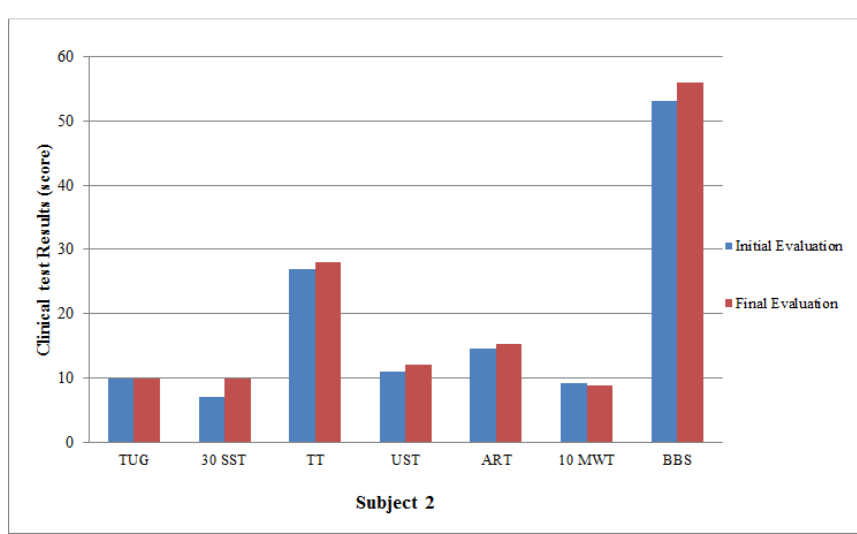

Fig. 3. Clinical test Results of subject 2 using the ABAR system.

\section{DISSCUSSION AND CONCLUSIONS}

Both patients improved clinical test results at the end of all sessions and the follow-up, with improvements in the ART (8.67 $\mathrm{cm}$ in Subject 2), in BBS (from 41 to 53 in Subject 1) and TT (from 23 to 26 in Subject 1). Finally, the results obtained in the SEQ test reported high levels of satisfaction.

The purpose of this study, improvements in balance and gait, has been accomplished. Patients enjoyed the sessions and were motivated during VMR. However, due to the presence of residual deficits, it is necessary to continue using these therapies focusing on the balance, postural control, and gait rehabilitation. Future studies will be based on the design of new functionalities in the ABAR system to train dynamic balance.

\section{REFERENCES}

[1] A.H. Ropper "The Guillain-Barré syndrome," N Engl J Med, 326, pp. 1130-1136, 1992.

[2] R.A. Hughes and D.R. Cornblath, "Guillain-Barré syndrome," Lancet, 366(9497), pp. 1653-66, Review, 2005.

[3] J.J. Sejvar, A.L. Baughman, M. Wise and O.W. Morgan, "Population incidence of Guillain-Barré syndrome: a systematic review and metaanalysis," Neuroepidemiology, 36(2), pp. 123-133, 2011.

[4] Y.L. Lo, "Clinical and immunological spectrum of the Miller Fisher syndrome," Muscle Nerve, 36(5), pp. 615-27, 2007.

[5] E.K. Vanhoutte et al., "Modifying the Medical Research Council grading system through Rasch analyses," Brain, 135(5), pp. 1639-49, 2012.

[6] R.A. Hughes, E.F. Wijdicks, et al, "Supportive care for patients with Guillain-Barré syndrome," Arch Neurol, 62(8),pp.1194-8, 2005.

[7] J.M.Meythaler, "Rehabilitation of Guillain-Barré syndrome," Arch Phys Med Rehabil, 78(8), pp.872-9, 1997.

[8] C. White, J. Pritchard and L. Turner-Stokes, "Exercise for people with peripheral neuropathy," Cochrane Database Sist Rev, (4), 2004.

[9] Garssen MP et al., "Physical training and fatigue, fitness and quality of life in Guillain-Barre Syndrome and CIPP," Neurology, 63(12), pp. 2393-5, 2004

[10] K.H. Pitetti, P.J. Barrett and D. Abbas, "Endurance exercise training in Guillain-Barre Syndrome,” Arch Phys Med Rehabil, 74(7), pp. 761-5, 1993.

[11] H. Weiss, V. Rastan, W. Müllges, R.F. Wagner and K.V. Toyka, “ Psychotic symptoms and emotional distress in patients with GuillainBarré syndrome," Eur Neurol, 47(2), pp.74-8, 2002.
[12] A. Fosberg, R. Press, U. Einarsson, et al. "Impairment in Guillain-Barre síndrome during the first 2 years after onset: a prospective study," J Neurol Sci, 227(1), pp. 131-138, 2004.

[13] C. Bauer, I. Gröger, R. Rupprecht and K.G. Gassmann, "Intrasession reliability of force platform parameters in community-dwelling older adults," Arch Phys Med Rehabil, 89(10), pp. 1977-82, 2008.

[14] B. Lange, S. Koenig, C.Y. Chang, E. McConnell, E. Suma, M. Bolas and A. Rizzo, "Designing Informed Game-Based Rehabilitation Tasks Leveraging Advances in Virtual Reality,” Disability and Rehabilitation, vol. 34, pp. 1863-70, 2012.

[15] M.S. Cameirão, S.B. Badia, E.D. Oller and P.F. Verschure, "Neurorehabilitation using the virtual reality based Rehabilitation Gaming System: methodology, design, psychometrics, usability and validation,” J Neuroeng Rehabil, vol. 7, 2010.

[16] A. Rizzo, G. Jounghyun Kim, "A SWOT analysis of the field of virtual reality rehabilitation and therapy", Presence: Teleoper. Virtual Environ, 14(2), pp. 119-146, 2005.

[17] S. Albiol-Pérez, J.A. Gil-Gómez, M. Alcañiz, R. Llorens, and C. Colomer. "Use of the Wii balance board system in vestibular rehabilitation," 13th International Conference on Interacción PersonaOrdenador (INTERACCION '12), 2012.

[18] J.D. Holmes, M.E. Jenkins, A.M. Johnson, M.A. Hunt and R.A.Clark, "Validity of the Nintendo Wii(R) balance board for the assessment of standing balance in Parkinson's disease," Clin Rehabil, 2012.

[19] J.A. Gil-Gómez, R. Lloréns, M. Alcañiz and C. Colomer, "Effectiveness of a Wii balance board-based system (eBaViR) for balance rehabilitation: a pilot randomized clinical trial in patients with acquired brain injury," J Neuroeng Rehabil, vol. 23, 2011.

[20] S. Albiol-Pérez, J.A. Lozano-Quilis, H. Gil-Gómez, J.A. Gil-Gómez, R. Llorens, "Virtual rehabilitation system for people with Parkinson's disease," 9th international conference on disability, virtual reality and associated technologies (ICDVRAT), pp. 423-427, 2012.

[21] M.F. Folstein, S.E. Folstein and P.R. Mchugh, "Mini-Mental State: a practical method for grading the cognitive state of patients for the clinicians," J Psychiatr Res, vol. 12, pp. 189-198, 1975.

[22] C. Collin, D.T. Wade, S. Davies and V. Horne, "The Barthel ADL Index: a reliability study," Int Disabil Stud, vol 10, pp. 61-3, 1988.

[23] M.P. Lawton, "The Philadelphia Geriatric Center Morale Scale: a revision," J Gerontol, vol 30, pp. 85-9, 1975.

[24] M.E. Charlson, P. Pompei, K.L. Ales and C.R. MacKenzie, "A new method of classifying prognostic comorbidity in longitudinal studies: development and validation," J Chronic Dis, vol 40, pp. 373-83, 1987.

[25] G.P. Austin, D. Tiberio and G.E. Garrett, "Effect of frequency on human unipedal hopping," Percept Mot Skills, vol. 95, pp. 733-40, 2002.

[26] P.W. Duncan, D.K. Weiner, J. Chandler and S. Studensk,"Functional reach: a new clinical measure of balance," J Gerontol 1990, vol. 45, pp. 192-197, 1990.

[27] K.O. Berg and S.L. Wood-Dauphinee, J.I. Williams and B. Maki, "Measuring balance in the elderly: validation of an instrument," Can Jl of Public Health, vol. 83, pp. 7-11, 1992.

[28] D. Podsiadlo and S. Richardson, "The time "up \& go": a test of basic functional mobility for frail elderly persons," J Am Geriatr Soc, vol. 39, pp. 142-148, 1991.

[29] V.A. Scholtes et al., "Lower limb strength training in children with cerebral palsy-a randomized controlled trial protocol for functional strength training based on progressive resistance exercise principles," BMC Pediatr, 8, pp. 8:41, 2008.

[30] M.E. Tinetti, T.F. Williams and R. Mayewski, "Fall risk index for elderly patients based on number of chronic disabilities," Am J Med, 80(3), pp. 429-34, 1986.

[31] S. O'Shea, N. Taylor and J. Paratz, "Reliability of hand-held dynamometry and functional strength tests for the lower extremity in children with cerebral palsy," Arch Phys Med Rehabil, 88(1), pp. 32-36, 2007. 